Cite this: Phys. Chem. Chem. Phys. 2014, 16, 16755

Received 26th March 2014 Accepted 23rd June 2014

DOI: $10.1039 / c 4 c p 01311 \mathrm{e}$

www.rsc.org/pccp

\section{Energy transfer in aggregates of bacteriochlorophyll $c$ self-assembled with azulene derivatives $\dagger$}

\author{
Martina Matěnová, ${ }^{a}$ Viviana Lorelei Horhoiu, ${ }^{\text {bc }}$ Florian-Xuan Dang, ${ }^{d}$ Petr Pospíšil, ${ }^{a}$ \\ Jan Alster, ${ }^{a}$ Jaroslav V. Burda, ${ }^{a}$ Teodor Silviu Balaban ${ }^{\text {bcd }}$ and Jakub Pšenčík ${ }^{\star a}$ \\ Bacteriochlorophyll $(\mathrm{BChl}) \mathrm{c}$ is the main light-harvesting pigment of certain photosynthetic bacteria. It is \\ found in the form of self-assembled aggregates in the so-called chlorosomes. Here we report the \\ results of co-aggregation experiments of $\mathrm{BChl} c$ with azulene and its tailored derivatives. We have \\ performed spectroscopic and quantum chemical characterization of the azulenes, followed by self- \\ assembly experiments. The results show that only azulenes with sufficient hydrophobicity are able to \\ induce aggregation of $\mathrm{BChl} c$. Interestingly, only azulene derivatives possessing a conjugated phenyl ring \\ were capable of efficient ( 50\%) excitation energy transfer to $\mathrm{BChl}$ molecules. These aggregates \\ represent an artificial light-harvesting complex with enhanced absorption between 220 and $350 \mathrm{~nm}$ \\ compared to aggregates of pure $\mathrm{BChl} c$. The results provide insight into the principles of self-assembly \\ of $\mathrm{BChl}$ aggregates and suggest an important role of the $\pi-\pi$ interactions in efficient energy transfer.
}

\section{Introduction}

Bacteriochlorophyll $c(\mathrm{BChl} c)$ is similar to BChls $d$ and $e$, all three being found in chlorosomes, the light-harvesting antennae of photosynthetic bacteria of the phylum Chlorobi, some Chloroflexi, and the only one known Acidobacteria species. ${ }^{1,2}$ BChls $c, d$ and $e$ in chlorosomes are self-assembled into aggregates without any protein scaffold. The close proximity of BChl molecules in the aggregate leads to strong exciton coupling and consequently to a red shift of the $\mathrm{Q}_{\mathrm{y}}$ absorption band compared to the monomer. The aggregates form curved layers which are arranged in lamellar structures by a hydrophobic interaction between esterifying alcohols from neighboring layers. ${ }^{3-6}$ Tubular models also abound in the literature. ${ }^{1,6,7}$ Carotenoids and quinones are present in the chlorosomes as well, and located in the hydrophobic space between the lamellar layers. ${ }^{8}$ Such an arrangement ensures a close contact between these molecules and BChls without affecting excitonic coupling between BChls. Carotenoids serve for light-harvesting and photoprotective functions while

\footnotetext{
${ }^{a}$ Faculty of Mathematics and Physics, Charles University, Ke Karlovu 3, 12116 Prague, Czech Republic. E-mail: psencik@karlov.mff.cuni.cz

${ }^{b}$ Institute for Nanotechnology, Karlsruhe Institute for Technology (KIT), Postfach 3640, D-76021 Karlsruhe, Germany

${ }^{c}$ Center for Functional Nanostructures, KIT, Postfach 3640, D-76021 Karlsruhe, Germany

${ }^{d}$ Aix Marseille Université, Centrale Marseille, CNRS UMR 7313, Institut des Sciences Moléculaires de Marseille, Chirosciences, Marseille, France

$\dagger$ Electronic supplementary information (ESI) available: Synthesis of azulene derivatives A2-A4 and calculation of the azulene spectra. See DOI: 10.1039/ c4cp01311e
}

quinones are involved in the protective excitation quenching in the presence of oxygen. ${ }^{1}$ The orderly arrangement of pigments in domains inside the chlorosomes allows for efficient lightharvesting and excitation energy transfer. ${ }^{6,9}$ In addition, aggregation increases the stability against photodegradation compared to monomeric BChls. ${ }^{10}$

The ability of BChls $c, d$ and $e$ to self-assemble and their high light-harvesting efficiency make the BChl aggregates a promising material for artificial light-harvesting antennae. ${ }^{11,12}$ Both chlorosomal pigments and their synthetic analogs are intensively studied in this respect. ${ }^{12-20} \mathrm{BChl} c$ forms aggregates in organic nonpolar solvents and also in aqueous environments. In the latter case, addition of a suitable nonpolar compound is necessary to induce the aggregation. Other constituents of the chlorosomes, e.g. lipids, carotenoids and quinones, were shown to induce such aggregation in vitro. ${ }^{21-23}$ Each of these additional components also affects the properties of the formed complexes in a distinctive way. This ability can be used to prepare artificial complexes with tailored properties. For instance, if the aggregation is induced by pigment molecules absorbing light at the wavelengths where the BChl $c$ aggregates absorb poorly, it is possible to prepare artificial light-harvesting complexes with extended spectral coverage compared to pure BChl aggregates. ${ }^{24}$

In order to prepare an antenna combining BChls with another molecule, it is necessary to understand the nature of the involved interactions. The aggregation in the polar environment of the aqueous buffers is driven by a hydrophobic effect. $^{22,25}$ In addition, it has been suggested that $\pi-\pi$ or $\mathrm{CH}-\pi$ interactions play an important role in maintaining the close contact between carotenoids and BChls required for 
A
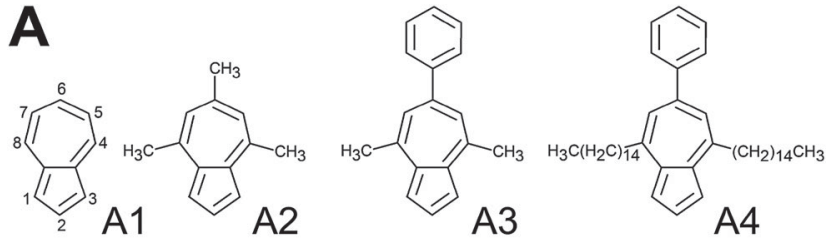

B
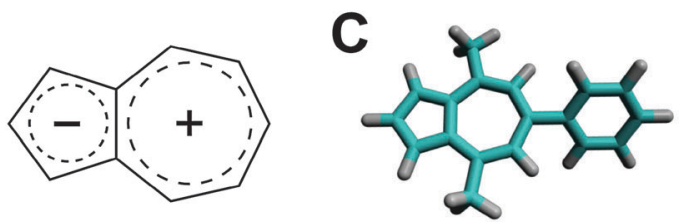

Fig. 1 (A) Chemical structure of azulene (A1) and its three derivatives (A2-A4) used in this work. (B) Schematic representation of the charge distribution within the azulene skeleton. (C) The optimized geometry of azulene A3

efficient energy transfer and photoprotection. ${ }^{26,27}$ To further investigate the role of the above-mentioned interactions in the co-aggregation with BChl $c$, we have studied the aggregationinducing properties of several azulene derivatives which have different substituents, a conjugated phenyl ring and/or hydrophobic side chains.

Azulene (Fig. 1) is an aromatic $10 \pi$ conjugated molecule related to naphthalene. It consists of fused cyclopentadiene and cycloheptatriene rings. Its skeleton is naturally found in some mushrooms and plants. ${ }^{28-30}$ It is a textbook example of a compound violating Kasha's rule and exhibiting fluorescence from the $\mathrm{S}_{2}$ state. $^{31}$ Azulene and its derivatives represent the first group of chromophores not found in chlorosomes, for which the ability to induce $\mathrm{BChl} c$ aggregation was investigated. Use of the series of derivatives differing in their side groups was instrumental in distinguishing between the role of the hydrophobicity, which is important for induction of BChl $c$ aggregation, and the role of the $\pi-\pi$ interactions, which seems to be critical for efficient transfer of excitation energy absorbed by azulenes to BChls. The derivatives containing a conjugated phenyl ring effectively extended the spectral coverage of the final assemblies into the near UV spectral region.

\section{Experimental methods}

Azulene was purchased from Sigma-Aldrich (99\%, A97203) and its three derivatives were synthesized by using Hafner's condensation of pyrylium salts ${ }^{32,33}$ with the sodium salt of cyclopentadiene (Scheme 1). ${ }^{34}$ Full experimental details are

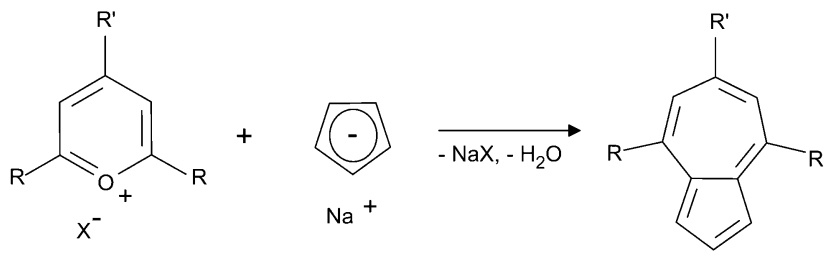

Scheme $1 \quad$ A2 R $=\mathrm{R}^{\prime}=\mathrm{CH}_{3} ; \mathrm{A} 3 \mathrm{R}=\mathrm{CH}_{3}, \mathrm{R}^{\prime}=\mathrm{C}_{6} \mathrm{H}_{5} ; \mathrm{A} 4 \mathrm{R}=-\left(\mathrm{CH}_{2}\right)_{14} \mathrm{CH}_{3}$, $\mathrm{R}^{\prime}=\mathrm{C}_{6} \mathrm{H}_{5}$. presented in the ESI. $\dagger$ For the numbering of pigments used within this work, see Fig. 1.

The hydrophobicity of the azulenes was calculated as $\log P$ defined here as the partition coefficient between octanol and water using Molinspiration software (www.molinspiration.com).

The optimized geometry of the azulene molecules was determined by quantum chemical calculation using the B3PW91 functional and the $6-31 G^{*}$ basis set within the Gaussian 09 software. ${ }^{35}$ The optical spectra were calculated by several methods based on both molecular orbital (MO) formalism and the density functional theory (DFT) method (see ESI $\dagger$ ). The most successful approach, the results of which are presented here, was the TDDFT/ $\omega$-B97-XD functional with aug-cc-pVDZ basis sets as implemented in a Gaussian programme package. ${ }^{35}$ Analysis of transition integrals between the corresponding molecular orbitals was performed using Molpro 2012.1 MATROP - matrix operation utility. ${ }^{36,37}$

Extinction coefficients of azulene and its derivatives were determined from absorption spectra. An amount of pigment between 1 and $5 \mathrm{mg}$ was weighed and then dissolved in tetrahydrofuran (THF) in a $5 \mathrm{ml}$ volumetric flask. This stock solution was diluted in a defined way and the absorbance at the absorption maximum in a $1 \mathrm{~cm}$ cuvette was determined using a Specord 250 spectrophotometer (Analytik Jena). Extinction coefficients were calculated from at least 10 samples according to the Lambert-Beer law.

BChl $c$ was isolated from the whole cells of Chlorobaculum (Cba.) tepidum and purified by HPLC as described before ${ }^{22}$ with the following modifications: an Eclipse XDB-C18 column has been used and the mobile phase and the flow rate were, respectively, $100 \%$ methanol, $1.5 \mathrm{ml} \mathrm{min}^{-1}$ during the first 25 minutes and $80 \%$ methanol and $20 \% n$-hexane, $2 \mathrm{ml} \mathrm{min}^{-1}$ for the next 15 minutes. All four well-resolved main BChl $c$ homologues found in Cba. tepidum were then combined together to imitate the natural distribution of $\mathrm{BChl} c$ in chlorosomes.

Self assembly experiments were performed in the following way. First, stock solutions of chromophores were prepared. BChl $c$ was dissolved in methanol and its concentration was determined using an extinction coefficient of $70 \mathrm{mM}^{-1} \mathrm{~cm}^{-1}$ at the $\mathrm{Q}_{\mathrm{y}}$ maximum. ${ }^{38}$ Azulene was dissolved in THF and the extinction coefficient determined in this study was used to adjust the concentration. The stock solutions were mixed together to reach the required molar ratio and rapidly injected into $3 \mathrm{ml}$ of stirred $50 \mathrm{mM}$ Tris-HCl buffer, $\mathrm{pH}$ 8.0. The amount of injected organic solvents did not exceed $1 \%(\mathrm{v} / \mathrm{v})$. The final concentration of BChl $c$ was approximately $20 \mu \mathrm{M}$ in all samples. Molar ratios of the azulene derivatives to $\mathrm{BChl} c$ ranged from $0.01: 1$ to $2: 1$. Aggregate formation was evaluated from the red shift of the BChl $c \mathrm{Q}_{\mathrm{y}}$ absorption band. Absorption spectra were measured immediately after preparation and after three days, when the aggregates were fully developed. It should be noted that the reported molar ratios were determined for initial mixtures. It is possible that a small fraction of the azulene molecules was not incorporated into the BChl $c$ aggregates and precipitated in the buffer.

Energy transfer from azulenes to BChl $c$ was examined by means of steady-state fluorescence spectroscopy. Fluorescence 
excitation spectra were recorded on a FluoroMax-2 fluorescence spectrometer (Jobin Yvon Spex). A 715 nm cut-off filter (Roper Scientific) was used to attenuate the stray light contribution and eliminate the emission of azulene as well as the transmission of the second order of the excitation light. The excitation spectra were corrected for the wavelength dependence of the excitation light intensity as described previously. ${ }^{39}$ Fluorescence spectra were recorded at least three days after the preparation of aggregates in order to have fully developed samples. The molar ratios of azulene to $\mathrm{BChl} c$ in aggregates for excitation spectra measurement ranged from $0.3: 1$ to $2: 1$, and absorbance of the sample was less than 0.2 in the BChl $c$ Qy maximum to minimize the effect of re-absorption or self-quenching. Excitation energy transfer efficiency was determined from a comparison of absorption $(1-T)$ and fluorescence excitation spectra. Since aggregate formation generally increases light scattering, absorption spectra used for comparison with the excitation spectra were corrected for the scattering using the method of Latimer and Eubanks. ${ }^{40,41}$

\section{Results}

\subsection{Extinction coefficient of azulenes}

Fig. 2 shows the absorption spectra of the four azulenes used in this work. The spectra show a weak $S_{0} \rightarrow S_{1}$ transition between 500 and $650 \mathrm{~nm}$, and $\mathrm{S}_{0} \rightarrow \mathrm{S}_{2}$ transition at around $340 \mathrm{~nm}$ for azulene $\mathbf{A 1}$, at $350 \mathrm{~nm}$ for $\mathbf{A 2}$ and at approximately $370 \mathrm{~nm}$ for the $\mathbf{A} \mathbf{3}$ and $\mathbf{A} 4$ derivatives. The strongest $S_{0} \rightarrow S_{3}$ transition is at $276 \mathrm{~nm}$ for azulene $\mathbf{A 1}$, at $287 \mathrm{~nm}$ for $\mathbf{A 2}$ and at approximately $300 \mathrm{~nm}$ for derivatives $\mathbf{A} 3$ and A4. The absorption maxima of azulenes $\mathbf{A} 3$ and $\mathbf{A 4}$ which possess the 6-phenyl ring, are thus more red shifted. This is an unexpected observation, since the conjugated system of the phenyl ring is partially decoupled from the conjugated system of azulene: the geometry optimization of azulene $\mathbf{A 3}$ shows that the plane of the 6-phenyl ring forms a dihedral angle of $\sim 50^{\circ}$ with the plane of the azulene moiety (Fig. 1C). The appendage of the optically transparent hydrocarbon chains (A3 $\rightarrow$ A4) leads to a much less pronounced red shift (from $300 \mathrm{~nm}$ to $304 \mathrm{~nm}$ at the main absorption band). The spectra of $\mathbf{A 3}$ and $\mathbf{A 4}$ exhibit a similar shape, which further indicates that the side chains do not affect the absorption properties of the molecules significantly. Upon increasing the number of carbon atoms in side chains the vibrational fine structure of the bands becomes less resolved, most probably due to conformational averaging (Fig. 2). Extinction coefficients of azulene and its derivatives in THF determined from absorption measurements are summarized in Table 1.

\subsection{Calculations of the azulene spectra}

The observed changes in extinction coefficients between the azulene derivatives are rather difficult to understand, considering that the conjugated backbone seems to be very similar in all explored molecules. To shed light on the quantum mechanical basis for these differences, electronic spectra were calculated using several methods based on both MO formalism and DFT.

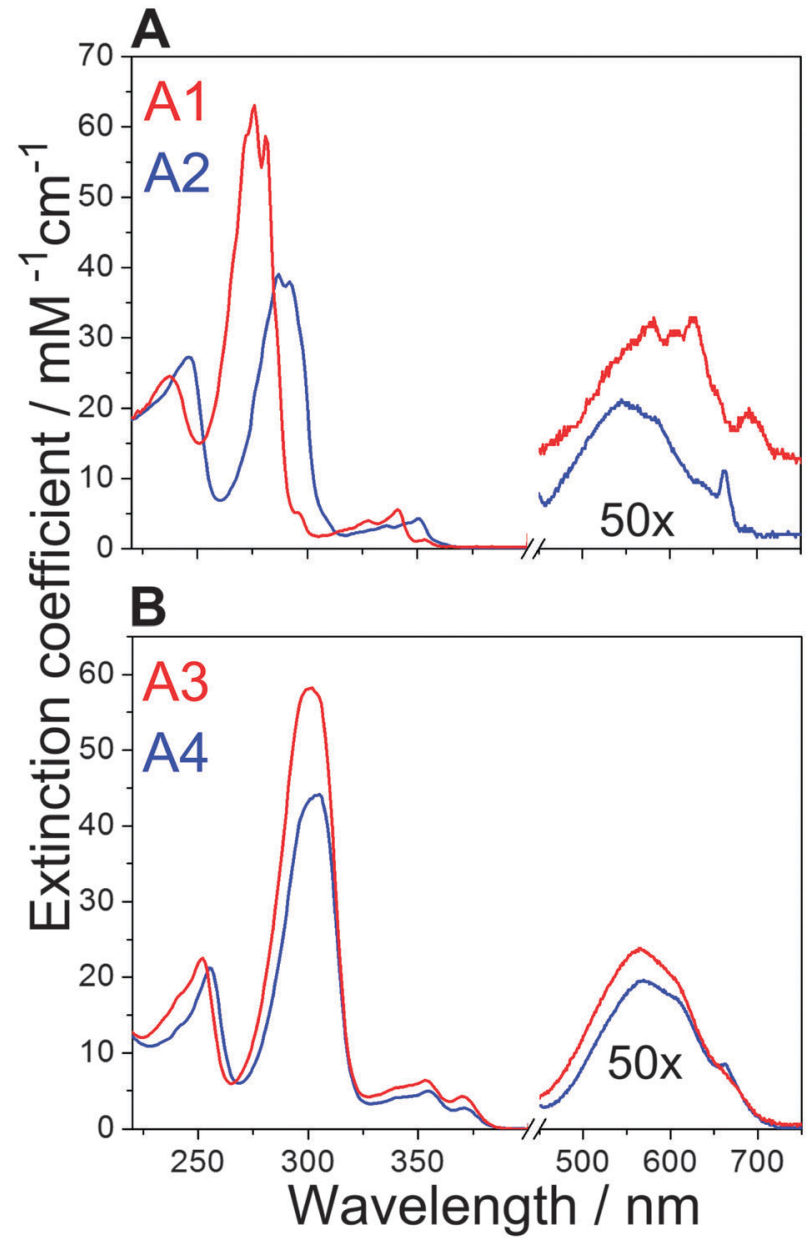

Fig. 2 Absorption spectra of azulene and its derivatives. (A) Absorption spectra of $\mathbf{A} \mathbf{1}$ (red) and $\mathbf{A} \mathbf{2}$ (blue). (B) Absorption spectra of A3 (red) and A4 (blue). Note the break in the $X$-axis and the change in scale.

Table 1 Properties of azulene A1 and its derivatives A2-A4

\begin{tabular}{lllr}
\hline Compound & $\begin{array}{l}\text { Absorption maximum } \\
\text { in THF }[\mathrm{nm}]\end{array}$ & $\begin{array}{l}\text { Extinction coefficient } \\
\text { in THF }\left[\mathrm{mM}^{-1} \mathrm{~cm}^{-1}\right]\end{array}$ & $\log P^{a}$ \\
\hline A1 & 276 & $63 \pm 3.0$ & 3.169 \\
A2 & 287 & $39 \pm 2.3$ & 4.371 \\
A3 & 301 & $58 \pm 2.4$ & 5.717 \\
A4 & 304 & $44 \pm 2.1$ & 10.539
\end{tabular}

${ }^{a}$ The partition coefficient between octanol and water.

The order of the states and their oscillator strengths roughly agree with experiment only when calculated using "long range corrected density functionals" (in our case TDDFT/ $\omega$-B97-XD and CAM-B3LYP functional methods ${ }^{42,43}$ ) except that these methods include one additional state with a very small oscillator strength. To be consistent with the convention used for azulenes in the literature where $S_{2}$ is used for the state from which fluorescence occurs ${ }^{31,44}$ and $S_{3}$ for the state with the maximal oscillator strength, ${ }^{45}$ we denote the transition with a negligible oscillator strength obtained from calculations as $\mathrm{S}_{0} \rightarrow \mathrm{S}_{x}$. More importantly, the obtained results quantitatively correctly describe the experimentally observed differences 
Table 2 Wavelengths and oscillator strengths $f$ for the first six transitions of A1-A4 calculated at the TDDFT/ $\omega$-B97-XD level

\begin{tabular}{|c|c|c|c|c|}
\hline \multirow[b]{2}{*}{ Transition } & \multicolumn{2}{|l|}{$\mathbf{A 1}$} & \multicolumn{2}{|l|}{$\mathbf{A 2}$} \\
\hline & Wavelength [nm] & $f$ & Wavelength [nm] & $f$ \\
\hline $\mathrm{S}_{0} \rightarrow \mathrm{S}_{1}$ & 517.9 & 0.008 & 412.3 & 0.027 \\
\hline $\mathrm{S}_{0} \rightarrow \mathrm{S}_{x}$ & 332.5 & 0.003 & 313.5 & 0.000 \\
\hline $\mathrm{S}_{0} \rightarrow \mathrm{S}_{2}$ & 260.8 & 0.073 & 261.4 & 0.300 \\
\hline $\mathrm{S}_{0} \rightarrow \mathrm{S}_{3}$ & 247.6 & 1.121 & 243.6 & 0.678 \\
\hline $\mathrm{S}_{0} \rightarrow \mathrm{S}_{4}$ & 236.7 & 0.000 & 232.7 & 0.000 \\
\hline \multirow[t]{2}{*}{$\mathrm{S}_{0} \rightarrow \mathrm{S}_{5}$} & 220.1 & 0.001 & 214.5 & 0.000 \\
\hline & \multicolumn{2}{|l|}{ A3 } & \multicolumn{2}{|l|}{ A4-Butyl } \\
\hline Transition & Wavelength [nm] & $f$ & Wavelength [nm] & $f$ \\
\hline $\mathrm{S}_{0} \rightarrow \mathrm{S}_{1}$ & 505.2 & 0.012 & 506.4 & 0.016 \\
\hline $\mathrm{S}_{0} \rightarrow \mathrm{S}_{x}$ & 342.2 & 0.025 & 342.4 & 0.012 \\
\hline $\mathrm{S}_{0} \rightarrow \mathrm{S}_{2}$ & 275.5 & 0.061 & 275.6 & 0.085 \\
\hline $\mathrm{S}_{0} \rightarrow \mathrm{S}_{3}$ & 272.9 & 1.384 & 274.6 & 1.292 \\
\hline $\mathrm{S}_{0} \rightarrow \mathrm{S}_{4}$ & 242.1 & 0.003 & 235.3 & 0.000 \\
\hline $\mathrm{S}_{0} \rightarrow \mathrm{S}_{5}$ & 238.3 & 0.000 & 230.2 & 0.002 \\
\hline
\end{tabular}

between the intensity of the strongest transition $S_{0} \rightarrow S_{3}$ (Table 2). Its oscillator strength is comparable for azulenes A1 and $\mathbf{A 3}$, but reduced approximately to one half for $\mathbf{A 2}$. The oscillator strength of this transition for azulene $\mathbf{A 4}$ is lower than that of A3. The difference between intensities of the $S_{0} \rightarrow S_{3}$ transition for azulenes $\mathbf{A} \mathbf{1}$ and $\mathbf{A} \mathbf{2}$ is even in a good quantitative agreement. In addition, the results obtained for azulene A1 agree well with previous studies ${ }^{46,47}$ and the minor differences are most probably caused by the different basis sets used. Because geometry optimization and electronic spectra calculation of the whole $\mathbf{A 4}$ molecule are computationally very demanding, the $\mathrm{C} 15$ aliphatic chains were replaced with ethyl, propyl, and butyl groups in order to examine the effect of the side chain length on the electronic spectra. The calculations revealed that the length of the side chain has almost no effect on the transition energies, but does have a small influence on the oscillator strengths. The calculated transition energies of A3 and A4 are thus very similar, in agreement with the experimental data (Table 2). Calculations where THF was included as an implicit solvent exhibit similar trends.

To gain a better understanding of the observed differences in extinction coefficients, the role of different molecular orbitals in the main electronic transitions was analyzed. Generally, the HOMO $\rightarrow$ LUMO excitation dominates in the $\mathrm{S}_{0} \rightarrow \mathrm{S}_{1}$ transition, however, its intensity is low. Next transition (between 310 and $350 \mathrm{~nm}$ ) corresponds almost equally to HOMO $-1 \rightarrow$ LUMO and HOMO $\rightarrow$ LUMO +1 excitations and its intensity is close to a dark state. DFT calculations further predict that the third transition is predominately represented by the HOMO - $1 \rightarrow$ LUMO +1 excitation. Its energy was calculated to be around $260-270 \mathrm{~nm}$ which is distinctly higher than that of the $S_{0} \rightarrow S_{1}$ and $S_{0} \rightarrow S_{x}$ transitions. The most intense spectral line for all of the explored azulenes corresponds to the fourth excited state. Its energy is close to the third transition (within $5-20 \mathrm{~nm}$ ), but the intensity is more than an order of magnitude higher in comparison with all previous transitions. To explain different intensities of the
$\mathrm{S}_{0} \rightarrow \mathrm{S}_{3}$ transition for different azulene derivatives, two effects have to be taken into account: the composition of the excited state and the values of molecular transition integrals $\left\langle\Psi_{x}|r| \Psi_{y}\right\rangle$. First, the fourth transition is a combined excitation of HOMO $1 \rightarrow$ LUMO and HOMO $\rightarrow$ LUMO +1 , similarly to the second transition. However, detailed analysis of the transition integrals between the corresponding MOs revealed that the $\angle$ HOMO $1|r| \mathrm{LUMO}\rangle$ and $\langle$ HOMO $|r| \mathrm{LUMO}+1\rangle$ integrals add up. This is in contrast to the second transition, in which the $\langle$ HOMO $1|r|$ LUMO $\rangle$ and $\langle$ HOMO $|r|$ LUMO +1$\rangle$ integrals cancel each other. The second effect is connected with the fact that for $\mathbf{A 2}$ the $\mathrm{S}_{0} \rightarrow \mathrm{S}_{3}$ transition contains some additional non-negligible admixtures of the $\langle$ HOMO $-x|r|$ LUMO $+y\rangle$ type (where at least one of $x, y>1$ ) with low integral values. Consequently the weights of the $\langle$ HOMO $-1|r|$ LUMO $\rangle$ and $\langle$ HOMO $|r|$ LUMO +1$\rangle$ integrals are decreased reducing the final transition intensity of this transition to approximately one-half of the value for A1.

\subsection{Solubility of azulenes in water}

It was proposed that the aggregation of $\mathrm{BChl}$ molecules in an aqueous environment is driven by the hydrophobic effect, and therefore the hydrophobicity of the aggregation-inducing molecule is expected to be an important parameter. The calculated hydrophobicities of azulenes A1-A4 are listed in Table 1. As can be expected already from the chemical structure of the molecules, the hydrophobicity increases from A1 through A2 and A3 to A4. This order was further confirmed by reverse phase HPLC chromatography and also by calculation of the solubility of azulenes in water (not shown). In agreement with all these estimates, we could observe that azulene $\mathbf{A 1}$ is partly soluble in water. Its solubility was previously determined to be $0.02 \mathrm{~g} \mathrm{l}^{-1}$ (ref. 48), which corresponds to a $160 \mu \mathrm{M}$ solution. The concentration of azulene A1 used for self-assembly experiments in this work was much lower. We have tested the solubility of all the azulenes in water. In all cases, the amount of azulene which corresponded to an intended final concentration of about $40 \mu \mathrm{M}$ was injected into $3 \mathrm{ml}$ of buffer. Absorption spectra were recorded after preparation and after three days to determine the degree of precipitation of azulene derivatives. The amount of azulenes in buffer decreased from $\mathbf{A 1}$ to A4. Azulene A1 remained dissolved in its original concentration, while azulenes A2, A3 and A4 precipitated to concentration of $\sim 20 \mu \mathrm{M}, \sim 10 \mu \mathrm{M}$ and $\sim 3 \mu \mathrm{M}$, respectively. The concentration was determined using the same extinction coefficient as in THF.

\subsection{Self-assembly experiments}

$\mathrm{BChl} c$ is partly soluble in water where it develops into a form whose absorption spectrum resembles that determined for an antiparallel dimer in dichlormethane. ${ }^{49}$ The $\mathrm{Q}_{\mathrm{y}}$ absorption maximum of this form is between 710 and $715 \mathrm{~nm}$ and we denote it as a dimer in this work. We use the term aggregate for assemblies in which the $\mathrm{Q}_{\mathrm{y}}$ band is distinctively red shifted above $715 \mathrm{~nm}$.

The ability of all azulenes to induce aggregation of BChl $c$ was examined by measuring steady-state absorption spectra upon mixing with an increasing concentration of azulene or its derivatives. 
The concentrations of azulene derivatives necessary to induce a red shift of the BChl $c \mathrm{Q}_{\mathrm{y}}$ band above $715 \mathrm{~nm}$ were then compared. If the aggregation is driven by the hydrophobic effect, as previously proposed, the aggregation inducing ability should increase from A1 through A2 and A3 to A4. This is exactly what we have observed. The results are summarized in Fig. 3. Azulene A1 was used in concentrations at which it is fully soluble in water $(<40 \mu \mathrm{M})$ and was not able to induce any aggregation even at the highest used molar ratio of $\mathbf{A 1}$ to BChl $c$ (2:1). The maximum of the BChl $c \mathrm{Q}_{\mathrm{y}}$ band in the sample containing azulene $\mathbf{A 1}$ and $\mathrm{BChl} c$ in a molar ratio of 2:1 was found to be at $\sim 715 \mathrm{~nm}$. The increase in the amount of azulene in the sample can be monitored by the intensity of the azulene absorption between 250 and $400 \mathrm{~nm}$. The total absorption spectrum thus most probably represents just a sum of spectra of azulene A1 and BChl $c$ dissolved in buffer. Azulene A2 induces aggregation only at the highest tested molar ratio of azulene to BChl $c$. For a molar ratio of azulene $\mathbf{A} 2$ to BChl $c$ of $1: 1$ the $\mathrm{Q}_{\mathrm{y}}$ maximum was observed at $\sim 719 \mathrm{~nm}$, while for the molar ratio of $2: 1$, the $\mathrm{Q}_{\mathrm{y}}$ band shifted to $\sim 730 \mathrm{~nm}$. This shift has to be caused by the interaction of BChl $c$ species with azulene A2.

The aggregation-inducing ability, however, improves significantly upon appending the 6-phenyl ring. Very similar results were obtained from experiments with both $\mathbf{A 3}$ and A4. Fig. 3C shows the absorption of BChl $c$ upon mixing with azulene $\mathbf{A 4}$. Addition of $\mathbf{A 4}$ to the sample leads to a significant red shift of the $\mathrm{BChl} c \mathrm{Q}_{\mathrm{y}}$ band, indicating pronounced aggregation of $\mathrm{BChl}$ $c$. The red shift increases with an increase of azulene concentration in the sample, which can be again monitored by the intensity of the azulene bands between 250 and $400 \mathrm{~nm}$. The Soret band of BChl $c$ is also affected by the aggregation, but to a lesser degree. The molar ratio between azulene $\mathbf{A 4}$ and BChl $c$ required to obtain the $\mathrm{Q}_{\mathrm{y}}$ band at a longer wavelength than $715 \mathrm{~nm}$ is about $0.1: 1$. The $\mathrm{Q}_{\mathrm{y}}$ band position of samples with the molar ratio of $0.1: 1$ was at $\sim 710 \mathrm{~nm}$ after preparation, but it shifted to $\sim 720 \mathrm{~nm}$ after aggregates fully developed within three days. The maximal red shift of $\sim 745 \mathrm{~nm}$ was observed for the highest molar ratio used $(2: 1)$. Azulene A3, which lacks the long hydrocarbon chains, was only slightly less efficient in inducing the aggregation of $\mathrm{BChl} c$. The molar ratio of $\mathbf{A} 3$ to $\mathrm{BChl} c$ required to induce a red-shift up to $715 \mathrm{~nm}$ is $0.2: 1$. The maximal red shift of the $\mathrm{BChl} c \mathrm{Q}_{\mathrm{y}}$ band was similar to azulene A4 at $\sim 745 \mathrm{~nm}$ and observed for the molar ratio of $2: 1$.

We can conclude that the absorption of azulene $\mathbf{A 1}$ observed in Fig. 3A after three days comes from the pigment dissolved in buffer. On the other hand, samples where azulenes A2-A4 induced aggregation of BChl $c$ exhibit substantially higher intensity of azulene absorption than what corresponds to their solubility in water, and the azulenes do not precipitate. Quantitative estimation of the azulene concentration embedded in the aggregate structure is difficult due to the contribution of BChl $c$ absorption and light-scattering at a given wavelength, and the necessity to use the extinction coefficient of azulene determined in THF. Nevertheless reasonable estimates were obtained. For instance, concentration of azulene $\mathbf{A 4}$ in the
A

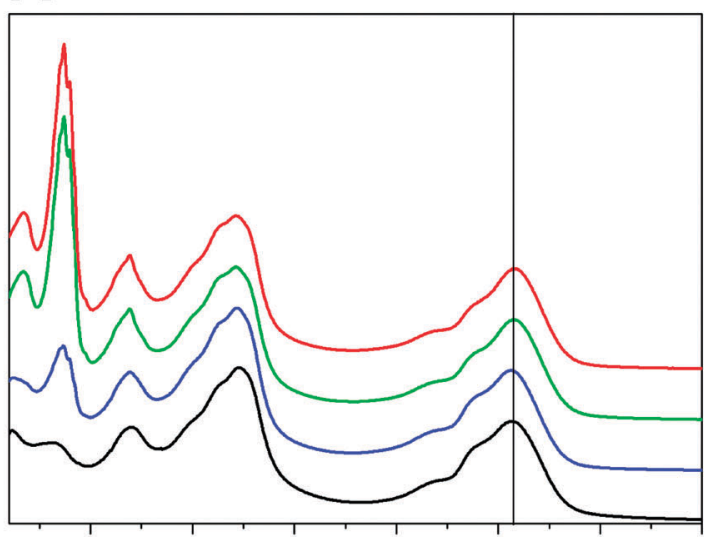

B

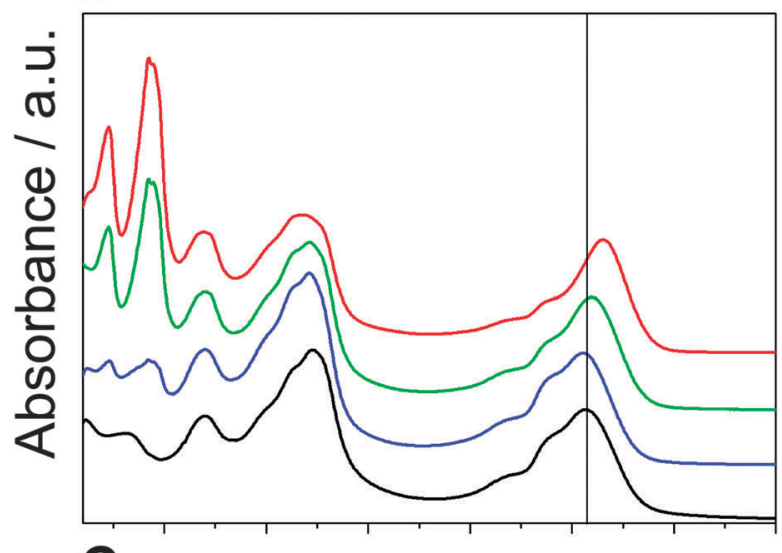

C

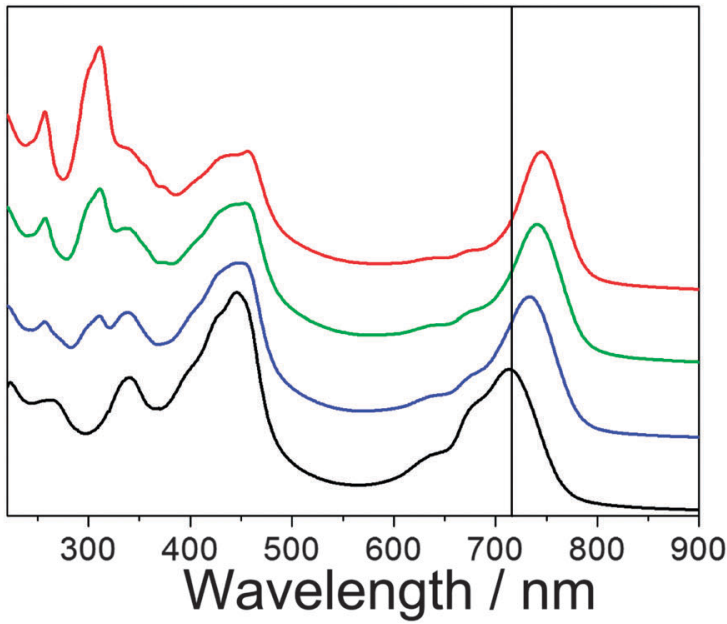

Fig. 3 Absorption spectra of BChl $c$ with A1 (A), A2 (B) and A4 (C) measured three days after preparation. The azulene to $\mathrm{BChl} C$ molar ratio is $0: 1$ (black), $0.3: 1$ (blue), $1: 1$ (green) and $2: 1$ (red). A vertical line at $715 \mathrm{~nm}$ is shown to assist the comparison of the $B C h l c Q_{y}$ band positions. Spectra were normalized at the maximum of $Q_{y}$ band and offset along the absorbance axis for the sake of clarity. Results for $\mathbf{A} \mathbf{3}$ are very similar to those for A4

sample prepared from a mixture of this azulene and BChl $c$ in a molar ratio of $2: 1$ was $\sim 20 \mu \mathrm{M}$, i.e. $6-7$ times higher than its solubility. This fact certainly means that the hydrophobic 
interaction leading to the aggregate formation leads also to incorporation of the azulene molecules within the aggregate structure. In addition, the absorption spectra of the red shifted BChl-azulene assemblies exhibit substantially larger scattering contribution which indicates that the aggregation leads to formation of much larger particles than the dimers as can be seen in Fig. 3C for azulene A4. Similar results were also observed for azulene A3 (not shown). This is in contrast to what we observed for azulene A1 where any significant contribution of the scattering was absent (Fig. 3A).

\subsection{Excitation energy transfer from azulenes to BChl $c$}

Steady-state fluorescence excitation spectroscopy was used to check whether the excitation energy can be transferred from the incorporated azulenes to $\mathrm{BChl} c$. The fluorescence quantum yield of BChl $c$ aggregates is certainly lower than that of the monomers, and thus does not represent the aggregate induced fluorescence, a phenomenon described and recently explained by Tang and coworkers. ${ }^{50}$ However, the quantum yield was high enough to allow determination of the energy transfer efficiency with a sufficient accuracy. Excitation spectra were recorded for samples with an azulene-to-BChl $c$ ratio of at least $0.3: 1$. For lower azulene-to-BChl $c$ ratios, the contribution of azulenes to the absorption spectrum was too weak to allow an accurate determination of the efficiency. The excitation spectra were detected close to the maximum of BChl $c$ emission (755 to $780 \mathrm{~nm}$ depending on the aggregation state of BChl c). The emission of azulene with a maximum at $\sim 420 \mathrm{~nm}$ (not shown) was eliminated using a cut-off filter. All measured excitation spectra exhibit spectral features of BChl $c$ in the same proportions as in the absorption spectra which indicates that internal conversion and vibrational relaxation within BChl $c$ occurs with close to $100 \%$ efficiency, as expected. In addition, the main absorption peak of azulene at $\sim 300 \mathrm{~nm}$ is discernible in the spectra from samples containing azulene $\mathbf{A 3}$ or $\mathbf{A 4}$, although its intensity does not correspond to that observed in the $(1-T)$ absorption spectra (Fig. 4). This means that only a part of the energy absorbed by azulene is transferred to BChl $c$. To determine the extent of the transferred energy, the excitation spectra were compared with absorption $(1-T)$ spectra of the sample as a reference for $100 \%$ efficiency. ${ }^{51,52}$ The efficiency of energy transfer was determined by comparing the intensity of $1-T$ and fluorescence excitation spectra at $\sim 260 \mathrm{~nm}$, where the absorption of azulene is negligible, and near $\sim 300 \mathrm{~nm}$, where it is maximal. The estimated energy transfer efficiencies vary with the concentration of the azulene in the sample. For both azulenes A3 and A4 the highest efficiency was determined for aggregates with an azulene to $\mathrm{BChl} c$ molar ratio of around 1:1 ( $\sim 50 \%$ for $\mathbf{A 3}$ and $\sim 60 \%$ for $\mathbf{A 4}$ ). In aggregates with a higher molar ratio of azulene, the energy transfer efficiency approximately linearly decreases $(\sim 40 \%$ efficiency for a molar ratio of azulene $\mathrm{A4}$ to BChl $c$ of $1.5: 1$; $\sim 30 \%$ efficiency for a molar ratio of $2: 1$ ). For a lower $\mathbf{A} 3$ or $\mathbf{A 4}$ to $\mathrm{BChl} c$ ratio $(0.3-0.8: 1)$ the energy transfer efficiency was estimated to be similar to that observed for $0.8-1.0: 1$ concentration.

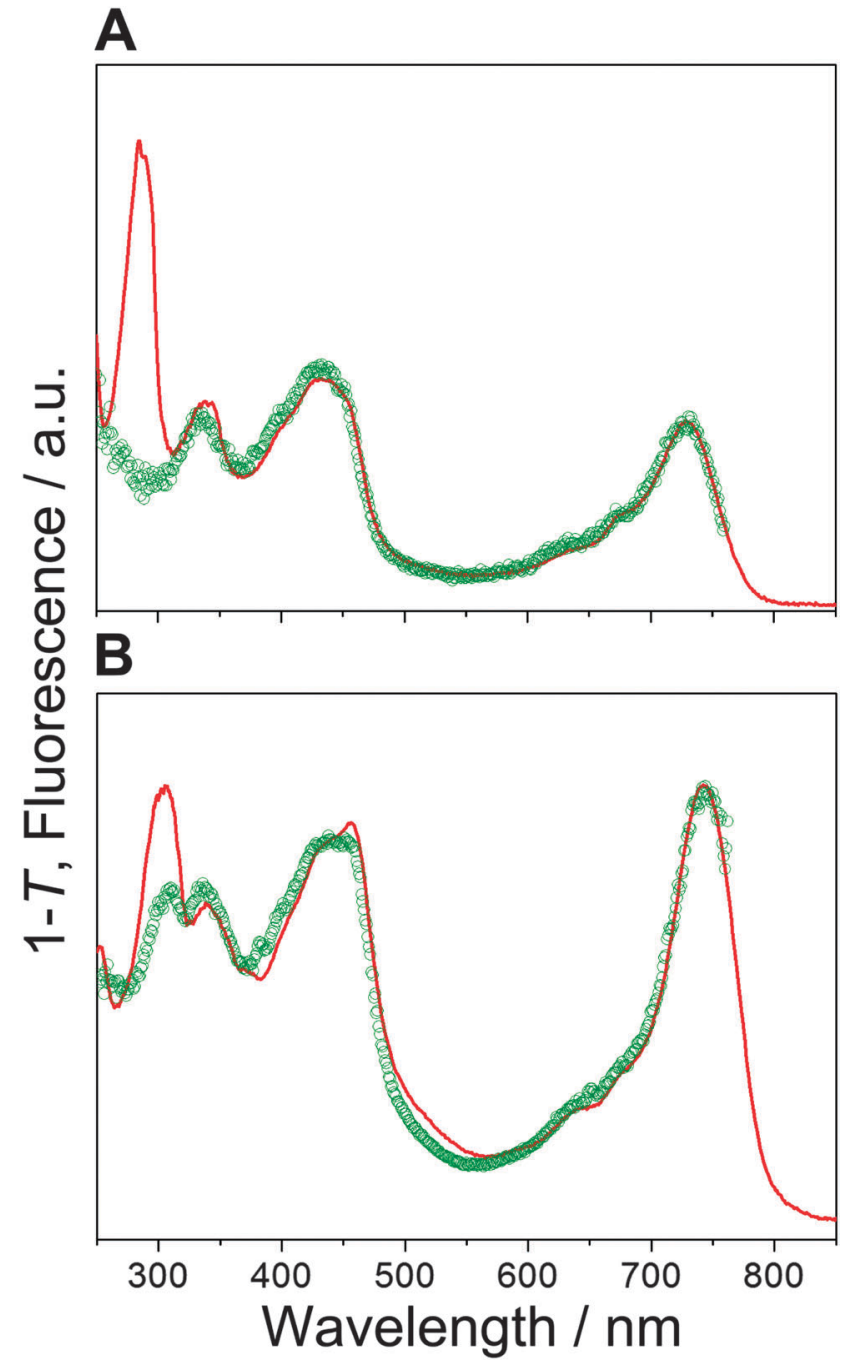

Fig. 4 Comparison of the fluorescence excitation spectrum (green circles) and the $1-T$ absorption spectrum (red) of $\mathbf{A} 2-B C h l ~ C$ aggregates (molar ratio $2: 1$ ) (A) and $\mathbf{A} 3-\mathrm{BChl} C$ aggregates (molar ratio 1:1) (B).

In contrast, the excitation spectra recorded for mixtures of BChl $c$ with azulenes $\mathbf{A 1}$ and $\mathbf{A 2}$ did not reproduce any features of the azulene absorption. Most importantly, no sign of energy transfer was observed even for those samples where the concentration of azulene A2 was high enough to induce aggregation of BChl $c$ (Fig. 4A). Thus, it can be safely concluded that these pigments do not transfer their excitation energy to BChl $c$ molecules even if they are incorporated in the aggregate structure.

\section{Discussion}

Self-assembly of chlorosomal BChls in aqueous environments induced by other nonpolar molecules naturally found in chlorosomes (lipids, carotenoids, quinones) has been well studied before. $^{12,21-23}$ These authors established that the hydrophobic interaction between the esterifying alcohols is not strong enough to drive the aggregation of $\mathrm{BChl} c$ molecules in aqueous environments. However, a nonpolar compound which is not 
soluble in water, but is soluble in a solvent miscible with water may induce aggregation. This is in contrast to the situation in nonpolar solvents, like hexane, where the hydrophilic interaction between the polar chlorin rings drives the interaction without the need for any additional molecule. To further characterize the properties of a molecule necessary to induce aggregation of BChl $c$ in aqueous environments, we have chosen to investigate azulene and its three derivatives studied in this work. Neither azulene nor its derivatives are found in chlorosomes, however, their easy synthesis via the pyrylium route and versatility in modification of the structure and properties make them a valuable model system.

The results show that an increase in hydrophobicity of the azulene molecule increases its ability to induce aggregation. Azulene A1 is partly soluble in water and is not able to induce aggregation even at the highest concentration used. On the other hand, both azulenes with a phenyl ring (A3 and A4) induce aggregation with a similar efficiency, with $\mathbf{A 4}$ being only slightly more efficient. This is interesting, because originally we assumed that the presence of long hydrophobic chains of $\mathbf{A 4}$ is required for aggregation induction. However, the hydrophobicity required to induce aggregation is clearly achieved already for azulene A3, and the effect of the additional hydrophobic side chains (A4 vs. A3) is rather weak although still discernible. These results prove that a certain hydrophobicity of the co-aggregating molecule is sufficient to induce aggregation, regardless of whether it is reached by incorporation of nonpolar chains or not.

Importantly, the results also show that even if the hydrophobicity of the chromophore molecules is high enough to induce aggregation of BChls, it does not necessarily ensure that the energy absorbed by a molecule incorporated in the aggregate will be transferred efficiently to BChls. This was observed for azulene $\mathbf{A 2}$, which is able to induce aggregation if the molar ratio of $\mathbf{A} 2$ to $\mathrm{BChl} c$ is larger than $1: 1$, but even in this case we did not observe any energy transfer from azulene $\mathbf{A} 2$ to $\mathrm{BChl} c$. In contrast, both $\mathbf{A} \mathbf{3}$ and $\mathbf{A 4}$ are able to transfer the absorbed excitation energy rather efficiently to BChl $c$ molecules. Consequently, these two azulene derivatives extend the spectral coverage of the prepared assemblies into the near UV region (200-350 nm). The observed energy transfer, together with the aggregation inducing ability of these two azulene derivatives, is an indication of a strong interaction between azulene and BChl $c$ molecules. This inference is further justified by the fact that azulenes A3 and A4 have very low solubility in water and precipitate in aqueous buffer, but not in assemblies with BChl $c$. The interaction between azulenes and BChls leading to BChl aggregate formation, and the persistence of azulenes in spectra, strongly indicate that azulenes $\mathbf{A 3}$ and $\mathbf{A 4}$ are incorporated in the aggregate structure.

The efficiency of ET is approximately constant up to a molar ratio of azulenes $\mathbf{A} 3$ and $\mathbf{A 4}$ to $\mathrm{BChl} c$ of $\sim 1: 1$, but then decreases with increasing azulene concentration. This is probably caused by excess of azulene molecules incorporated in the aggregates. Not all azulene molecules are then in close contact with the conjugated system of the BChl molecules and thus they are not able to transfer absorbed energy. Excitation energy

\section{Azulene BChl c}

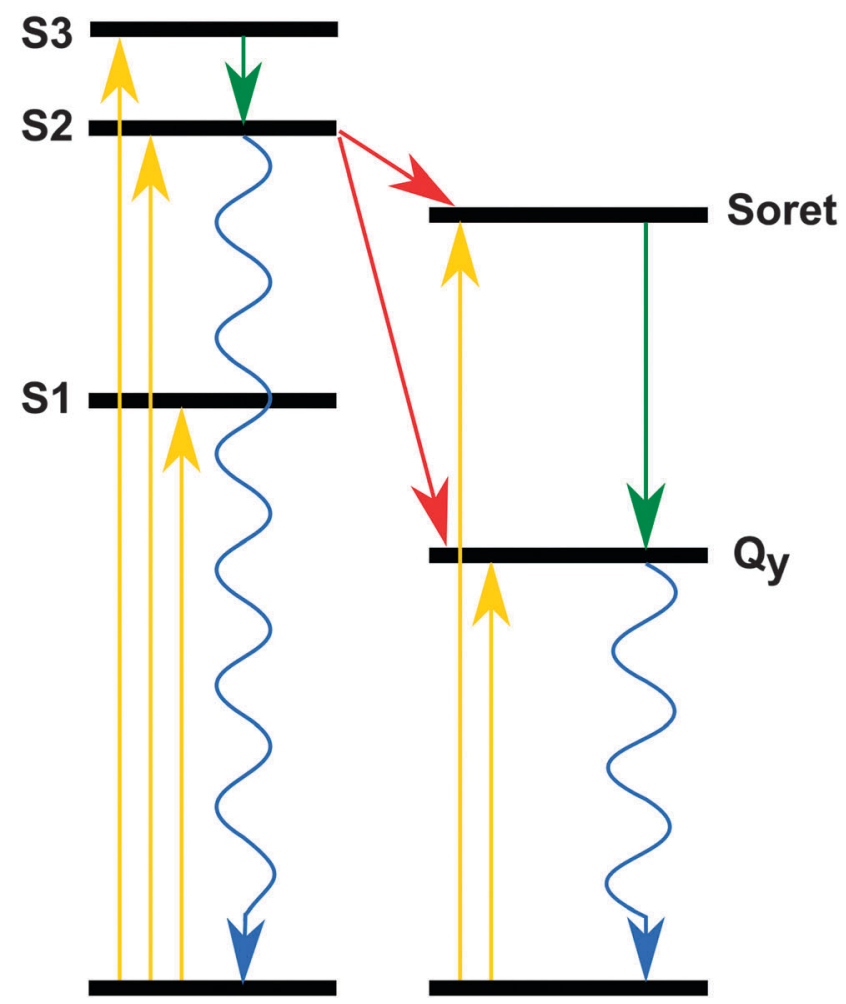

Fig. 5 Scheme of the energy levels and the most relevant transitions in the aggregates of $\mathrm{BChl} C$ with azulenes $\mathrm{A} 3$ and $\mathbf{A 4}$. Vertical arrows represent absorption (yellow) and internal relaxation (green), oblique arrows represent proposed energy transfer pathways (red) and wavy arrows (blue) represent fluorescence. Only the three lowest allowed states of azulene are shown for clarity.

transfer from azulene to $\mathrm{BChl} c$ occurs most probably from the $\mathrm{S}_{2}$ state of the azulene molecule to the Soret or $\mathrm{Q}_{\mathrm{y}}$ band of the $\mathrm{BChl} c$, and not from the $\mathrm{S}_{1}$ state of azulene to the $\mathrm{Q}_{\mathrm{y}}$ state of BChl $c$ (Fig. 5). Internal relaxation from $\mathrm{S}_{2}$ to $\mathrm{S}_{1}$ was suggested to be slow due to the small cross section between potential energy curves of extremely separated levels. ${ }^{31}$ Therefore energy transfer needs to compete only with a relatively slow fluorescence, which originates also from the $\mathrm{S}_{2}$ state. ${ }^{53}$

Our results imply an important role of the phenyl substituent in efficient energy transfer. The phenyl ring can be involved in $\pi-\pi$ interactions between azulene and BChl molecules which may ensure the proximity of pigments required for sufficient interaction and efficient energy transfer. These results are in line with our recently proposed hypothesis concerning BChlcarotenoid interactions in chlorosomes. ${ }^{27}$ As there is no protein in the chlorosome interior which would maintain the optimal distance and orientation of the pigments, there must be some other way how self-assembly can ensure this. It was proposed that $\pi-\pi$ (or $\mathrm{CH}-\pi$ ) interactions between the end-group of a carotenoid and the conjugated system of a BChl molecule are responsible for strong interactions required for observed efficient energy transfer between carotenoids and BChls in chlorosomes. ${ }^{26,27}$ 


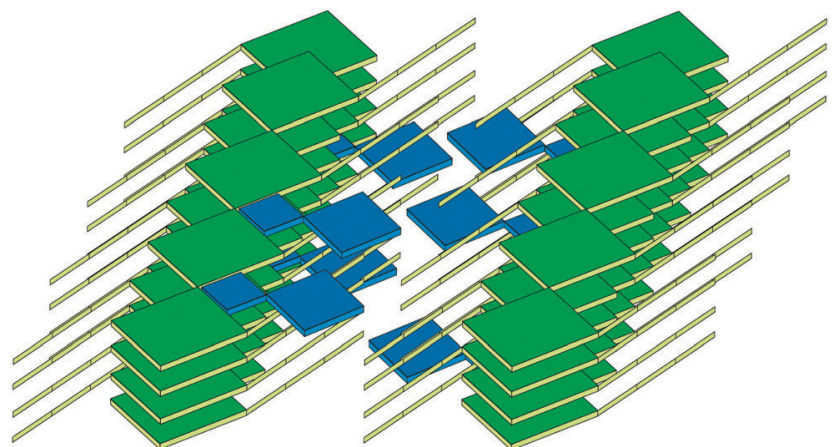

Fig. 6 Schematic representation of the proposed $\pi-\pi$ interaction between the phenyl group (smaller blue squares) of azulene A3 or A4 and the conjugated system of the $\mathrm{BChl}$ molecules (green squares with tails). The larger blue squares represent the azulene moiety.

It seems that azulenes without phenyl rings are not capable of such $\pi-\pi$ interactions with BChls. Both the cyclopentadiene and cycloheptatriene rings of the azulene molecule are components of the conjugated system that could potentially fit in the space available for interaction with the chlorin rings. ${ }^{27}$ However, the azulene $\mathbf{A 1}$ exhibits a permanent dipole moment of $1.08 \mathrm{D} .{ }^{54}$ To incorporate such a polar moiety is energetically unfavorable. In contrast, the phenyl group of azulenes A3 and A4 is well suited for $\pi-\pi$ interactions with the conjugated system of the BChl molecule (Fig. 6). We propose that the interaction between azulene and BChl molecules does not alter the hydrogen bonding pattern between the BChl molecules forming the aggregates. Instead, the phenyl ring is interacting with the part of the conjugated system of the BChl molecule, which is accessible from the side of the BChl layers. The proposed arrangement ensures a close interaction between azulene and $\mathrm{BChl}$ molecules required to explain the observed energy transfer without disrupting the excitonic coupling between BChls. Azulene A2, which induces aggregation at higher concentration but does not transfer energy, is probably also located in the hydrophobic space formed by esterifying alcohols of BChls from the two neighboring chlorin layers, but without any close interaction with the chlorin ring. Further study including quantum mechanical calculation and molecular modeling is required to verify or disprove this hypothesis, and is now underway.

In summary, this is the first study on incorporation of fully synthetic pigments with a spectral coverage different from the pure chlorosomal $\mathrm{BChl}$. It proves the functional construct, where in certain cases efficient excitation energy transfer occurs, and paves the way for applications in biomimetic dye aggregate solar cells. ${ }^{55}$

\section{Conclusions}

Results of co-aggregation experiments of BChl $c$ with azulene and three of its 4,6,8-trisubstituted derivatives provide further insight into the principles of self-assembly of BChl aggregates. They revealed that $\pi-\pi$ interactions mediated via the phenyl ring are important for the efficient energy transfer to $\mathrm{BChl} c$, and confirmed that a certain hydrophobicity of the molecule is required for the aggregation inducing ability of the admixed molecules, in this work azulenes. Once the aggregates are formed, the azulenes remain incorporated in the finally formed structures. Azulenes $\mathbf{A} \mathbf{3}$ and $\mathbf{A 4}$ are able to induce aggregation of BChl $c$ molecules and transfer the excitation energy absorbed between 220 and $350 \mathrm{~nm}$ to $\mathrm{BChl} c$. The formed complexes thus represent an artificial light-harvesting antenna with the spectral coverage extended into the near UV region compared to pure BChl $c$ aggregates. This study may open the way to light-harvesting devices with tailored properties.

\section{Acknowledgements}

This study was supported by Czech Science Agency, project No. P501/12/G055 and P208/12/0622. STB and VLH acknowledge financial support from the DFG through the Centre for Functional Nanostructures (CFN) within projects $\mathrm{C} 3.2$ and C3.6. The EU FP7 Future and Emerging Technologies (FET) Programme is thanked for the present generous funding of the Project PEPDIODE in Marseille.

\section{References}

1 N.-U. Frigaard and D. A. Bryant, in Complex Intracellular Structures in Prokaryotes, Microbiological Monographs, ed. J. Shively, Springer, Berlin, 2006, pp. 79-114.

2 D. A. Bryant, A. M. G. Costas, J. A. Maresca, A. G. M. Chew, C. G. Klatt, M. M. Bateson, L. J. Tallon, J. Hostetler, W. C. Nelson, J. F. Heidelberg and D. M. Ward, Science, 2007, 317, 523-526.

3 J. Pšenčík, T. P. Ikonen, P. Laurinmäki, M. C. Merckel, S. J. Butcher, R. E. Serimaa and R. Tuma, Biophys. J., 2004, 87, 1165-1172.

4 G. T. Oostergetel, M. Reus, A. G. M. Chew, D. A. Bryant, E. J. Boekema and A. R. Holzwarth, FEBS Lett., 2007, 581, 5435-5439.

5 J. Pšenčík, M. Torkkeli, A. Župčanová, F. Vácha, R. E. Serimaa and R. Tuma, Photosynth. Res., 2010, 104, 211-219.

6 G. T. Oostergetel, H. van Amerongen and E. J. Boekema, Photosynth. Res., 2010, 104, 245-255.

7 S. Ganapathy, G. T. Oostergetel, P. K. Wawrzyniak, M. Reus, G. M. A. Chew, F. Buda, E. J. Boekema, D. A. Bryant, A. R. Holzwarth and H. J. M. de Groot, Proc. Natl. Acad. Sci. U. S. A., 2009, 106, 8525-8530.

8 J. Pšenčík, J. B. Arellano, T. P. Ikonen, C. M. Borrego, P. A. Laurinmäki, S. J. Butcher, R. E. Serimaa and R. Tuma, Biophys. J., 2006, 91, 1433-1440.

9 J. Dostál, T. Mančal, R. Augulis, F. Vácha, J. Pšenčík and D. Zigmantas, J. Am. Chem. Soc., 2012, 134, 11611-11617.

10 H. Kim, H. Li, J. A. Maresca, D. A. Bryant and S. Savikhin, Biophys. J., 2007, 93, 192-201.

11 T. S. Balaban, Acc. Chem. Res., 2005, 38, 612-623. 
12 T. Miyatake and H. Tamiaki, Coord. Chem. Rev., 2010, 254, 2593-2602.

13 T. S. Balaban, in Handbook of Porphyrin Science with Applications to Chemistry, Physics, Materials Science, Engineering, Biology and Medicine, ed. K. Kadish, K. Smith and R. Guilard, Singapore, 2010, vol. 1, pp. 221-306.

14 S. Shoji, T. Hashishin and H. Tamiaki, Chem. - Eur. J., 2012, 18, 13331-13341.

15 S. Patwardhan, S. Sengupta, L. D. A. Siebbeles, F. Würthner and F. C. Grozema, J. Am. Chem. Soc., 2012, 134, 16147-16150.

16 V. Huber, S. Sengupta and F. Würthner, Chem. - Eur. J., 2008, 14, 7791-7807.

17 S. Sengupta, D. Ebeling, S. Patwardhan, X. Zhang, H. von Berlepsch, C. Böttcher, V. Stepanenko, S. Uemura, C. Hentschel, H. Fuchs, F. C. Grozema, L. D. A. Siebbeles, A. R. Holzwarth, L. Chi and F. Würthner, Angew. Chem., Int. Ed., 2012, 51, 6378-6382.

18 S. Sengupta and F. Wurthner, Acc. Chem. Res., 2013, 46, 2498-2512.

19 N. Takahashi, H. Tamiaki and Y. Saga, Tetrahedron, 2013, 69, 3638-3645.

20 V. B. Shah and P. Biswas, ACS Nano, 2014, 8, 1429-1438.

21 M. Hirota, T. Moriyama, K. Shimada, M. Miller, J. M. Olson and K. Matsuura, Biochim. Biophys. Acta, Bioenerg., 1992, 1099, 271-274.

22 P. Klinger, J. B. Arellano, F. Vácha, J. Hála and J. Pšenčík, Photochem. Photobiol., 2004, 80, 572-578.

23 J. Alster, A. Župčanová, F. Vácha and J. Pšenčík, Photosynth. Res., 2008, 95, 183-189.

24 J. Alster, T. Polívka, J. Arellano, P. Chábera and J. Pšenčík, Chem. Phys., 2010, 373, 90-97.

25 A. Župčanová, J. B. Arellano, D. Bína, J. Kopecký, J. Pšenčík and F. Vácha, Photochem. Photobiol., 2008, 84, 1187-1194.

26 M. Fuciman, P. Chábera, A. Župčanová, P. Hříbek, J. B. Arellano, F. Vácha, J. Pšenčík and T. Polívka, Phys. Chem. Chem. Phys., 2010, 12, 3112-3120.

27 J. Pšenčík, J. B. Arellano, A. M. Collins, P. Laurinmäki, M. Torkkeli, B. Löflund, R. E. Serimaa, R. E. Blankenship, R. Tuma and S. J. Butcher, J. Bacteriol., 2013, 195, 1727-1734.

28 S. Carret, A. Blanc, Y. Coquerel, M. Berthod, A. E. Greene and J.-P. Deprés, Angew. Chem., Int. Ed., 2005, 44, 5130-5133.

29 C. Zhang, H. Liang, G. Tu and Y. Zhao, Fitoterapia, 2010, 81, 849-851.

30 A. D. Harmon, K. H. Weisgraber and U. Weiss, Experientia, 1980, 36, 54-56.

31 M. Beer and H. C. Longuet-Higgins, J. Chem. Phys., 1955, 23, 1390-1391.

32 T. S. Balaban and A. T. Balaban, in Science of Synthesis Houben-Weyl, Methods of Molecular Transformations, ed. E. J. Thomas, Thieme, Stuttgart, 2003, vol. 14, pp. 11-200.

33 T. S. Balaban and A. T. Balaban, in Science of Synthesis Knowledge Updates, ed. E. J. Thomas and A. P. Dobbs, Thieme, 2013, vol. 3, pp. 145-216.
34 K. Hafner and H. Kaiser, Justus Liebigs Ann. Chem., 1958, 618, 140-152.

35 M. J. Frisch, G. W. Trucks, H. B. Schlegel, G. E. Scuseria, M. A. Robb, J. R. Cheeseman, G. Scalmani, V. Barone, B. Mennucci, G. A. Petersson, H. Nakatsuji, M. Caricato, X. Li, H. P. Hratchian, A. F. Izmaylov, J. Bloino, G. Zheng, J. L. Sonnenberg, M. Hada, M. Ehara, K. Toyota, R. Fukuda, J. Hasegawa, M. Ishida, T. Nakajima, Y. Honda, O. Kitao, H. Nakai, T. Vreven, J. A. Montgomery Jr., J. E. Peralta, F. Ogliaro, M. Bearpark, J. J. Heyd, E. Brothers, K. N. Kudin, V. N. Staroverov, R. Kobayashi, J. Normand, K. Raghavachari, A. Rendell, J. C. Burant, S. S. Iyengar, J. Tomasi, M. Cossi, N. Rega, N. J. Millam, M. Klene, J. E. Knox, J. B. Cross, V. Bakken, C. Adamo, J. Jaramillo, R. Gomperts, R. E. Stratmann, O. Yazyev, A. J. Austin, R. Cammi, C. Pomelli, J. W. Ochterski, R. L. Martin, K. Morokuma, V. G. Zakrzewski, G. A. Voth, P. Salvador, J. J. Dannenberg, S. Dapprich, A. D. Daniels, Ö. Farkas, J. B. Foresman, J. V. Ortiz, J. Cioslowski and D. J. Fox, GAUSSIAN 09, Gaussian, Inc., Wallingford CT, 2009.

36 H.-J. Werner, P. J. Knowles, G. Knizia, F. R. Manby, M. Schütz, P. Celani, T. Korona, R. Lindh, A. Mitrushenkov, G. Rauhut, K. R. Shamasundar, T. B. Adler, R. D. Amos, A. Bernhardsson, A. Berning, D. L. Cooper, M. J. O. Deegan, A. J. Dobbyn, F. Eckert, E. Goll, C. Hampel, A. Hesselmann, G. Hetzer, T. Hrenar, G. Jansen, C. Köppl, Y. Liu, A. W. Lloyd, R. A. Mata, A. J. May, S. J. McNicholas, W. Meyer, M. E. Mura, A. Nicklass, D. P. O’Neill, P. Palmieri, D. Peng, K. Pflüger, R. Pitzer, M. Reiher, T. Shiozaki, H. Stoll, A. J. Stone, R. Tarroni, T. Thorsteinsson and M. Wang, Molpro 2012.1, 2012.

37 H.-J. Werner, P. J. Knowles, G. Knizia, F. R. Manby and M. Schütz, Wiley Interdiscip. Rev.: Comput. Mol. Sci., 2012, 2, 242-253.

38 R. Y. Stanier and J. H. C. Smith, Biochim. Biophys. Acta, 1960, 41, 478-484.

39 J. Alster, T. Polívka, J. B. Arellano, P. Hříbek, F. Vácha, J. Hála and J. Pšenčík, Photosynth. Res., 2012, 111, 193-204.

40 K. R. Naqvi, M. N. Merzlyak and T. B. Melø, Photochem. Photobiol. Sci., 2004, 3, 132-137.

41 P. Latimer and H. C. A. Eubanks, Arch. Biochem. Biophys., 1962, 98, 274-285.

42 S. Hirata and M. Head-Gordon, Chem. Phys. Lett., 1999, 314, 291-299.

43 J.-D. Chai and M. Head-Gordon, J. Chem. Phys., 2008, 128, 084106.

44 T. Itoh, Chem. Rev., 2012, 112, 4541-4568.

45 N. Tetreault, R. S. Muthyala, R. S. H. Liu and R. P. Steer, J. Phys. Chem. A, 1999, 103, 2524-2531.

46 M. Dierksen and S. Grimme, J. Chem. Phys., 2004, 120, 3544-3554.

47 D. P. Chong, Can. J. Chem., 2010, 88, 787-796.

48 L. I. Sweet and P. G. Meier, Bull. Environ. Contam. Toxicol., 1997, 58, 268-274.

49 M. Umetsu, R. Seki, T. Kadota, Z.-Y. Wang, T. Adschiri and T. Nozawa, J. Phys. Chem. B, 2003, 107, 9876-9882. 
50 Y. Hong, J. W. Y. Lam and B. Z. Tang, Chem. Commun., 2009, 53 A. E. W. Knight and B. K. Selinger, Chem. Phys. Lett., 1971, 4332-4353. 12, 419-421.

51 R. Blankenship, Molecular Mechanism of Photosnythesis, 54 A. G. Anderson, Jr and B. M. Steckler, J. Am. Chem. Soc., Blackwell Science, 2002. 1959, 81, 4941-4946.

52 M. D. Yilmaz, O. A. Bozdemir and E. U. Akkaya, Org. Lett., 55 P. L. Marek, H. Hahn and T. S. Balaban, Energy Environ. Sci., 2006, 8, 2871-2873. 2011, 4, 2366-2378. 